TRANSACTIONS OF 'THE

AMERICAN MATHEMATICAL SOCIETY

Volume 285, Number 2, October 1984

\title{
GENERIC REDUCING FIELDS OF JORDAN PAIRS
}

BY

HOLGER P. PETERSSON

Max Koecher zur Vollendung des 60. Lebensjahres qewidmet

\begin{abstract}
Generic reducing fields of Jordan pairs, generalizing at the same time generic splitting fields of associative algebras and generic zero fields of quadratic forms, are intrinsically defined and constructed. The most elementary properties are derived, and the relationship with other generic constructions, particularly those linked to Brauer-Severi varieties, are investigated. As an application it is shown that there exist nonisomorphic exceptional Jordan division algebras having the same splitting fields.
\end{abstract}

After their first, though somewhat implicit, appearance in a paper by Witt [28], generic splitting fields of algebraic structures received extensive treatment in the setting of associative algebras by Amitsur $[\mathbf{1}]$ and Roquette $[\mathbf{2 3}, \mathbf{2 4}]$. Having been investigated further by Ferrar [5] for composition algebras and Knebusch [11, 12] for quadratic forms, the latter's point of view leading to new perspectives in the associative theory pursued by Heuser [7], Kovacs [13], and Saltman [25], Jacobson [8], appealing to the theory of Jordan algebras, recently made contributions along these lines to associative algebras with involution.

In the present paper, we go one step further and propose the theory of Jordan pairs as a general framework where a fair amount of the material mentioned above may be recovered in a unified fashion. It goes without saying that the sole justification of such an approach lies in a treatment which is intrinsic, i.e., which does not require the classification of finite-dimensional simple Jordan pairs. This is accomplished here while, at the same time, keeping the amount of "abstract" Jordan pair theory to comparatively modest proportions.

Among the various peculiarities which are typical for the point of view adopted here, the following is particularly important: In dealing with finite-dimensional Jordan structures, one has to distinguish carefully between the property of being reduced as compared to the one of being split. In fact, the former notion obviously being the more elementary of the two, it is clear that a first thrust into the subject must concern itself with reducing fields rather than splitting fields of Jordan pairs.

As a rule, all generic splitting fields known to date may conveniently be interpreted as rational function fields of appropriate algebraic varieties. The present paper provides no exceptions. In fact, we find it convenient to deduce a number of properties for generic reducing fields by making use of this interpretation, blaming everything on elementary results from algebraic geometry. To be more explicit, after having derived the most elementary properties in $\S 2$, we construct two classes of generic reducing fields, the first one being related, in the manner described above,

Received by the editors November 21, 1983.

1980 Mathematics Subject Classification. Primary 17C20; Secondary 10C10, 12F20, 16A46.

(C) 1984 American Mathematical Society $0002-9947 / 84 \$ 1.00+\$ .25$ per page 
to varieties of rank-one elements $(\S 3)$, the second one to generic norm surfaces $(\S 4)$. Specific examples are discussed in $\S 5$. In particular, we relate the generic reducing fields constructed before to generic zero fields of quadratic forms and generic splitting fields of associative algebras, with or without involution, most notably those attached to Brauer-Severi varieties. The paper concludes with an application to exceptional simple Jordan algebras arising from the first Tits construction (Theorem 6.1).

During the preparation of this work, the author benefited immensely from numerous conversations, accumulating over the years, he had with Professor J. C. Ferrar. To him he would like to express his sincere gratitude. It may be worthwhile to mention in closing that Professor Ferrar recently suggested the setting of linear algebraic groups as conceivably being the really appropriate framework for dealing with generic splitting fields.

1. Preliminaries. Let $k$ be a field of arbitrary characteristic. All vector spaces, algebras etc., with the possible exception of field extensions, are tacitly assumed to be finite-dimensional. Tensor products which do not specify the base ring are always to be taken over $k$. Scalar extensions will frequently be indicated by subscripts, e.g., $V_{l}$, for a vector space $V$ over $k$ and a field extension $l / k$, means the same as $V \otimes l$. Whenever invertibility makes sense in a structure $A$, the set of invertible elements in $A$ is denoted by $A^{\times}$. Given a quadratic mapping $Q: V \rightarrow W$ between vector spaces $V, W$, we invariably write $\left(v, v^{\prime}\right) \mapsto Q\left(v, v^{\prime}\right)$ for its bilinearization, so $Q\left(v, v^{\prime}\right)=Q\left(v+v^{\prime}\right)-Q(v)-Q\left(v^{\prime}\right)$ for $v, v^{\prime} \in V$.

Extensive use will be made of the theory of Jordan pairs, the basic reference being Loos [14], whose terminology we adopt throughout, mostly without further explanation. Just recall the definition of a Jordan pair over $k$ as a pair of $k$ vector spaces $V^{\varepsilon}, \varepsilon= \pm$, together with a pair of quadratic mappings $Q^{\varepsilon}: V^{\varepsilon} \rightarrow$ $\operatorname{Hom}_{k}\left(V^{-\varepsilon}, V^{\varepsilon}\right)$ such that, dropping superscripts whenever there is no danger of confusion and writing

$$
\{u v w\}=D(u, v) w=Q(u, w) v
$$

for $u, w \in V^{\varepsilon}, v \in V^{-\varepsilon}$, the following relations hold under all scalar extensions.

$$
\begin{aligned}
D(u, v) Q(u) & =Q(u) D(v, u), \\
D(Q(u) v, v) & =D(u, Q(v) u), \\
Q(Q(u) v) & =Q(u) Q(v) Q(u) .
\end{aligned}
$$

Throughout this paper we fix a Jordan pair $\mathcal{V}$ over $k$ which is absolutely simple in the sense that it stays simple in every base field extension. In addition to the material of [14], we also require a few results from [19]. Writing $\mathcal{V}_{i j}(X)(i, j \in \mathbf{Z})$ for the Peirce components of $\mathcal{V}$ relative to an orthogonal system $X$ of idempotents, we deduce from the Main Theorem, combined with its first corollary and Proposition 2 in $[\mathbf{1 9}]$, the following result.

Conjugacy TheOREM 1.1. Let $X=\left(c_{1}, \ldots, c_{r}\right)$ and $Y=\left(e_{1}, \ldots, e_{s}\right)$ be frames in $\mathcal{V}$. Then $r=s$, and there exists an inner automorphism $h$ of $\mathcal{V}$ satisfying $h V_{i i}(X)=V_{i i}(Y)$ for all $i=1, \ldots, r$.

Proposition 1.2 [19, Corollary 4 of the main theorem]. Given two maximal idempotents $e, e^{\prime}$ of $\mathcal{V}$, the subpairs $\mathcal{V}_{2}(e)$ and $\mathcal{V}_{2}\left(e^{\prime}\right)$ are conjugate under the inner automorphism group. 
LEMMA $1.3[19$, Lemma 4]. Let $X, Y$ be orthogonal systems of idempotents of the same length in $\mathcal{V}$ and $h$ an automorphism satisfying $h \mathcal{V}_{i i}(X)=\mathcal{V}_{i i}(Y)$ for all $i$. Then $h \mathcal{V}_{i j}(X)=\mathcal{V}_{i j}(Y)$ for all $i, j$.

We further require a number of standard results from algebraic geometry. To stress the elementary character of our approach, we adopt the classical point of view, due to Weil, and choose, once and for all, a huge universal domain $\Omega$ which is an algebraically closed extension field of $k$ of infinite transcendence degree. When it comes to rationality questions, our basic reference will be the introductory chapter of Borel [2]. Recall that a field extension $K / k$ is said to be regular in case it is separable and $k$ is algebraically closed in $K$. If this is so, and $L / k$ is any field extension, the free composite of $K, L$ (over $k$ ) is written as $K \cdot L$ (and defined to be the quotient field of $K \otimes L$ ). Natural examples of regular field extensions arise as rational function fields of $k$-varieties. More precisely, we record the following well-known facts.

Proposition 1.4. Let $X$ be an irreducible algebraic variety defined over $k$ and write $k(X)$ for the field of rational functions on $X$ defined over $k$ [2, AG (12.1)]. Then

(a) $k(X) / k$ is a regular field extension. Moreover, if we are given any field extension $l / k$, then $l(X)$, the field of rational functions on $X$ defined over $l$, is $l$-isomorphic to the free composite $k(X) \cdot l$.

(b) If $Y$ is a second irreducible algebraic variety defined over $k$, then $k(X \times Y)$ and $k(X) \cdot k(Y)$ are $k$-isomorphic.

(c) $X$ has $k(X)$-rational points.

Finally, we make use of the elementary theory of places. Here our basic reference, including matters of notation, is Bourbaki [3]. Accordingly, given field extensions $K / k, L / k$, we obtain sets $K^{\sim}, L^{\sim}$ by adjoining an additional symbol $\infty$ to $K, L$, respectively, and so a $k$-place (or a place over $k$ ) from $K$ to $L$ is the map $K^{\sim} \rightarrow L^{\sim}$ enjoying the usual properties, being, in particular, the identity on $k$. In addition, the following lemma will be useful [5, Lemma 2].

LEMMA 1.5. Let $k\left(X_{1}, \ldots, X_{n}\right)$ be the rational function field in $n$ indeterminants $X_{1}, \ldots, X_{n}, l / k$ a field extension and $\alpha_{1}, \ldots, \alpha_{n} \in l$. Then there exists $a$ $k$-place $\mathfrak{p}: k\left(X_{1}, \ldots, X_{n}\right)^{\sim} \rightarrow l^{\sim}$ satisfying $\mathfrak{p}\left(X_{i}\right)=\alpha_{i}$ for $1 \leq i \leq n$.

2. Reducing fields and places. Returning to the absolutely simple Jordan pair $\mathcal{V}$ over $k$ fixed in $\S 1$, an idempotent $c$ of $\mathcal{V}$ is said to be reduced in case it is nonzero and satisfies $\mathcal{V}_{2}(c)^{\varepsilon}=k c^{\varepsilon}$ for $\varepsilon= \pm$. We say $\mathcal{V}$ itself is reduced if it contains at least one reduced idempotent. Thanks to the Conjugacy Theorem 1.1, this implies that all local idempotents in $\mathcal{V}$ are reduced. The concept of a reduced Jordan pair naturally relates to elements of rank one, the rank of $u \in \mathcal{V}^{\varepsilon}$ being defined as the rank of the linear operator $Q(u): \mathcal{V}^{-\varepsilon} \rightarrow \mathcal{V}^{\varepsilon}$.

LEMMA 2.1. (a) Every element of $\mathcal{V}^{+}$extends to an idempotent, that is, given $u \in \mathcal{V}^{+}$, there is an idempotent $c=\left(c^{+}, c^{-}\right)$satisfying $c^{+}=u$. Moreover, if $u$ has rank one, every such $c$ is reduced.

(b) $\mathcal{V}$ is reduced if and only if $\mathcal{V}^{+}$contains elements of rank one.

Proof. (b) follows immediately from (a). To prove (a), let $u \in \mathcal{V}^{+}$. By von Neumann regularity $[\mathbf{1 4}, \mathbf{1 0 . 1 7}]$, some $v \in \mathcal{V}^{-}$satisfies $Q(u) v=u$, and $[\mathbf{1 4}, 5.2]$ 
shows that $c=(u, Q(v) u)$ is an idempotent of the desired kind. The last assertion of $(\mathrm{a})$ is obvious.

A field extension $K / k$ is called a reducing field of $\mathcal{V}$ in case the extended Jordan pair $V_{K}$ becomes reduced over $K$. Using our previous lemma, we can now show that the property of being a reducing field of $\mathcal{V}$ is preserved under places over $k$.

PROPOSITION 2.2. Let $K / k$ be a reducing field of $\mathcal{V}$ and $L / k$ an arbitrary field extension. If there exists a $k$-place from $K$ to $L$, then $L / k$ is a reducing field of $\mathcal{V}$ as well.

ProOF. Let $\mathfrak{p}: K^{\sim} \rightarrow L^{\sim}, \alpha \mapsto \bar{\alpha}$, be a $k$-place, with valuation ring $R$, and write $u \mapsto \bar{u}$ for the natural maps $\mathcal{V}^{\varepsilon} \otimes R \rightarrow \mathcal{V}^{\varepsilon} \otimes L$ induced by $\mathfrak{p}$. Following Lemma 2.1(b), we choose an element $u \in \mathcal{V}^{+}$of rank one. In order to show that $L$ reduces $\mathcal{V}$, there is no harm in assuming $\mathfrak{p}(R)=L$ and $u \in \mathcal{V}^{+} \otimes R-\mathcal{V}^{+} \otimes \mathfrak{m}, \mathfrak{m}$ being the maximal ideal of $R$. Now, as $u$ has rank one and is von Neumann regular, each $v \in \mathcal{V}^{-} \otimes R$ satisfies $Q(u) v=\rho u$ for some $\rho \in K$. The left-hand side living in $\mathcal{V}^{+} \otimes R$, this forces $\rho \in R$, hence $Q(\bar{u}) \bar{v}=\bar{\rho} \bar{u}$. Thus $\bar{u}$ has rank one, and Lemma 2.1(b) shows that $\mathcal{V} \otimes L$ is reduced, as claimed.

Proposition 2.2 naturally leads to the following definition, introducing a notion which is absolutely central for the entire paper.

DEFINITION 2.3. A generic reducing field of $\mathcal{V}$ is a field extension $K / k$ such that an arbitrary field extension $L / k$ reduces $\mathcal{V}$ if and only if there is a $k$-place from $K$ to $L$.

By General Abstract Nonsense, a generic reducing field, assuming it exists, is unique up to equivalence, where two extension fields of $k$ are said to be equivalent in case there are $k$-places in both directions. However, a generic reducing field is, of course, not unique up to $k$-isomorphism. In fact, we shall presently exhibit two elementary procedures yielding new generic reducing fields out of old ones. To begin with, let $e$ be a nontrivial idempotent of $\mathcal{V}$. Then standard arguments from the theory of Jordan pairs show that $\mathcal{W}=\mathcal{V}_{2}(e)$ makes up an absolutely simple subpair of $\mathcal{V}$ and $\mathcal{W}_{2}(c)=\mathcal{V}_{2}(c)$ for every idempotent $c$ in $\mathcal{W}$. Hence

Proposition 2.4. Let e be a nonzero idempotent in $\mathcal{V}$. Then $\mathcal{V}$ and $\mathcal{V}_{2}(e)$ have the same (generic) reducing fields.

Subsequently we shall construct "functions" assigning to each $\mathcal{V}$ a generic reducing field $K(\mathcal{V})$. Passing to $\mathcal{V}_{2}(e)$ as above, it follows from Proposition 2.4 that $K\left(\mathcal{V}_{2}(e)\right)$ is another generic reducing field of $\mathcal{V}$ which, in general, does not agree with $K(\mathcal{V})$. The second procedure may be found in the next proposition.

PROPOSITION 2.5. Let $K / k, L / K$ be field extensions and suppose $L / K$ is purely transcendental and finitely generated. Then $K / k$ is a generic reducing field of $\mathcal{V}$ if and only if $L / k$ is a generic reducing field of $\mathcal{V}$.

ProOF. The canonical imbedding $K \hookrightarrow L$ determines a $k$-place from $K$ to $L$, and Lemma 1.5 produces one in the reverse direction. Hence the field extensions $K / k$ and $L / k$ are equivalent, and the assertion follows.

In dealing with generic reducing fields, Proposition 2.4 allows us to shrink $\mathcal{V}$ to $V_{2}(e)$, for some idempotent $e$ of $\mathcal{V}$ (a maximal one, for example), hence to assume if necessary that $\mathcal{V}$ contains invertible elements. Then $\mathcal{V}$ has the form $\mathcal{V}=(J, J)$, where $J$ is an absolutely simple Jordan algebra over $k, \mathcal{V}^{+}=\mathcal{V}^{-}=J$ 
and $Q^{+}=Q^{-}$is the ordinary $U$-operator on $J$. Thus it seems that we just as well could have placed the entire set-up into the somewhat more elementary framework of Jordan algebras rather than that of Jordan pairs. However, as already becomes apparent from the proofs of our preceding results, most notably of Proposition 2.2, and will also follow from the subsequent development, it is the latter point of view as opposed to the former which is best suited for the problems which concern us here.

EXAMPLE 2.6. Let $A$ be a central simple associative algebra over $k$. Setting $\mathcal{V}^{+}=\mathcal{V}^{-}=A$, and defining a quadratic mapping $Q=Q^{+}=Q^{-}$from $A$ to $\operatorname{End}_{k}(A)$ by $Q(u) v=u v u$ for $u, v \in A$, we obtain an absolutely simple Jordan pair over $k$, written as $\mathcal{V}=\mathcal{V}(A)$ and called the Jordan pair associated with $A$. Clearly, $\mathcal{V}$ is reduced if and only if $A$ is split. Hence the (generic) reducing fields of $\mathcal{V}$ are the same as the (generic) splitting fields of $A$. Thus Proposition 2.2 implies the well-known fact that the property of being a splitting field of $A$ is preserved under places over $k$. Its proof, however, in particular when restricted to the special case considered here, is much more straightforward than the ones given in $[\mathbf{1}, \mathbf{2 3}, \mathbf{2 5}$. Now suppose $m$ is a positive integer and put $\mathcal{V}_{m}=\mathcal{V}\left(\mathrm{M}_{m}(A)\right)$, the Jordan pair associated with the algebra $\mathrm{M}_{m}(A)$ of $m \times m$ matrices having entries in $A$. Then there is an obvious way of choosing an idempotent $e$ in $\mathcal{V}_{m}$ satisfying $\mathcal{V}_{m 2}(e) \cong \mathcal{V}$, and Proposition 2.4 implies the trivial fact that the (generic) splitting fields of $A$ and $\mathrm{M}_{m}(A)$ agree. Starting from a single generic splitting field of $A$, this elementary procedure, phrasable in Jordan terms, yields a whole series of these, which has first been considered by Roquette [23].

EXAMPLE 2.7. Let $V$ be a vector space over $k$ of dimension $>2$ and $q: V \rightarrow k$ a quadratic form which is absolutely nondegenerate in the sense that in every base field extension the condition $q(v)=q(v, w)=0$ for all $w$ implies $v=0$. Setting $\mathcal{V}^{+}=\mathcal{V}^{-}=V$, and defining a quadratic mapping $Q=Q^{+}=Q^{-}$from $V$ to $\operatorname{End}_{k}(V)$ by $Q(v) w=q(v, w) v-q(v) w$ for $v, w \in V$, we obtain an absolutely simple Jordan pair over $k$, written as $\mathcal{V}=\mathcal{V}(q)$ and called the Jordan pair associated with $q$. It is well known and not hard to see that $\mathcal{V}$ is reduced if and only if the quadratic form $q$ is isotropic. Hence the (generic) reducing fields of $\mathcal{V}$ are the same as the (generic) zero fields of $q$ in the sense of Knebusch [11], where a field extension $K / k$ is defined to be a zero field of $q$ in case the scalar extension of $q$ to $K$ becomes isotropic. Thus Proposition 2.2 tells us that the property of being a zero field of $q$ is preserved under places over $k$. This is a well-known fact and also quite easy to prove directly. For a substantial generalization of this in characteristic $\neq 2$, see [11, Proposition 3.1].

3. The projective field of a Jordan pair. In this section, we wish to construct a generic reducing field of $\mathcal{V}$ which is closely related to elements of rank one. We write $T$ for the generic trace of $\mathcal{V}$ (so $T: \mathcal{V}^{+} \times \mathcal{V}^{-} \rightarrow k$ is a bilinear form which may be degenerate in characteristic two). Note that, thanks to the relations

$$
\begin{aligned}
T(\{u v w\}, x) & =T(w,\{v u x\}), \\
T(u, Q(v) w) & =T(w, Q(v) u), \\
T(Q(u) v, x) & =T(Q(u) x, v)
\end{aligned}
$$

for $u, w \in \mathcal{V}^{+}, v, x \in \mathcal{V}^{-}[14,16.8,16.12]$, the Peirce components of $\mathcal{V}$ relative to an idempotent $e$ are $T$-orthogonal (so $T\left(\mathcal{V}_{i}(e)^{+}, \mathcal{V}_{j}(e)^{-}\right)=0$ for integers $i \neq j$ ), 
and that $T$ restricted to $\mathcal{V}_{2}(e)$ yields the generic trace of $\mathcal{V}_{2}(e)$ [18, Lemma 6(b)]. The following elementary observation will be useful.

LEMMA 3.1. (a) An element $u \in \mathcal{V}^{+}$has rank one if and only if $u \neq 0$ and $Q(u) v=T(u, v) u$ for all $v \in \mathcal{V}^{-}$.

(b) Let $e$ be an idempotent of $\mathcal{V}$ and suppose $u \in \mathcal{V}^{+}$has rank at most one in $\mathcal{V}$. Then $u_{2}$, the Peirce-2-component of $u$ relative to $e$, has rank at most one in $V_{2}(e)$

(c) Suppose $\mathcal{V}$ contains invertible elements. Then to each $u \in \mathcal{V}^{+}$there exists an invertible element $y \in \mathcal{V}^{-}$satisfying $Q(u) y=u$.

ProOF. By Lemma 2.1(a), we may complete $u$ to an idempotent $c=\left(c^{+}, c^{-}\right)$, $c^{+}=u$, of $\mathcal{V}$ which is reduced in case $u$ has rank one.

(a) The condition is clearly sufficient. Conversely, let $u$ be of rank one and choose $c$ as above. Then the Peirce-2-component of $v \in \mathcal{V}^{-}$relative to $c$ has the form $\alpha c^{-}$, for some $\alpha \in k$, and taking traces yields $\alpha=T\left(c^{+}, v\right)$. Hence $Q(u) v=Q\left(c^{+}\right) \alpha c^{-}=\alpha c^{+}=T(u, v) u$.

(b) It is straightforward to check $Q\left(u_{2}\right) v=T\left(u_{2}, v\right) u_{2}$ for $v \in \mathcal{V}_{2}(e)^{-}$, so (a) applies. (By the way, the rank of $u_{2}$ in $\mathcal{V}_{2}(e)$ obviously agrees with its rank in $\mathcal{V}$.)

(c) With $c$ as above, we choose a frame $\left(c_{1}, \ldots, c_{r}\right)$ of $V_{2}(c)$ and extend it to a frame $\left(c_{1}, \ldots, c_{s}\right)(s \geq r)$ of $\mathcal{V}$. Then $e=\sum_{i=1}^{s} c_{i}$ is a maximal idempotent, so Proposition 1.2 implies $\mathcal{V}=\mathcal{V}_{2}(e)$, and $\mathcal{V}_{0}(c)=\mathcal{V}_{2}\left(\sum_{i>r} c_{i}\right)$ contains invertible elements. Choosing $y_{0} \in \mathcal{V}_{0}(c)^{-}$invertible, it then suffices to put $y=c^{-}+y_{0}$.

For char $k \neq 2$, Lemma 3.1(c) is due to Helwig-Hirzebruch [6, Satz 2]. Now, given a vector space $V$ over $k$, we write $\mathbf{P}_{k}(V)$ for the projective space $\mathbf{P}\left(V_{\Omega}\right)$, viewed canonically as an algebriac variety defined over $k$. For a nonzero vector $v \in V_{\Omega}$, denote by $[v]$ the line passing through $v$ and the origin.

THEOREM 3.2. The collection of rank one elements in $\mathcal{V}_{\Omega}^{+}$, i.e.,

$$
\operatorname{Proj}_{k}(\mathcal{V})=\left\{[u] \in \mathbf{P}_{k}(\mathcal{V}): u \in \mathcal{V}_{\Omega}^{+}, \operatorname{rank} u=1\right\}
$$

is an irreducible projective algebraic variety defined over $k$, called the projective variety of $\mathcal{V}$.

Proof. By Lemma 3.1(a), $X=\operatorname{Proj}_{k}(\mathcal{V})$ is closed in $\mathbf{P}_{k}\left(\mathcal{V}^{+}\right)$. Following $[\mathbf{1 4}$, 15.14], the inner automorphism group $G=\operatorname{Inn}\left(\mathcal{V}_{\Omega}\right)$ of $\mathcal{V}_{\Omega}$ is a connected algebraic group defined over $k$ which acts $k$-morphically on $\mathbf{P}_{k}\left(\mathcal{V}^{+}\right)$via $(\eta,[u]) \mapsto\left[\eta^{+} u\right]$, where $\eta=\left(\eta^{+}, \eta^{-}\right)$. Since every rank one element extends to a reduced idempotent (Lemma 2.1(a)), and every reduced idempotent extends to a frame, we obtain an induced action of $G$ on $X$ which, by the Conjugacy Theorem 1.1, is transitive, hence forces $X$ to be irreducible. Writing $k_{s}$ for the separable closure of $k$ in $\Omega$, we now contend that $X$ has $k_{s}$-rational points. Indeed, if $c$ is a division idempotent of $\mathcal{V}^{\prime}=\mathcal{V}_{k_{s}}$ and $d$ denotes the degree of $\mathcal{V}_{2}^{\prime}(c)$, then $\mathcal{V}_{2}^{\prime}(c)$ is the Jordan pair of a separable Jordan division algebra of degree $d$ over $k_{s}$. The latter must contain a separable (maximal) subfield of degree $d$, which forces $d=1$, hence that $V^{\prime}$ is reduced. Therefore $X$ does indeed contain $k_{s}$-rational points and, transitively being acted upon by $G$, must be defined over $k_{s}[2, \mathrm{AG}(14.5)]$. Clearly, by Lemma 3.1(a), $X\left(k_{s}\right)$ stays invariant under the Galois group of $k_{s} / k$, hence $[2, \operatorname{AG}(14.4)]$ implies that $X$ is defined over $k$.

We are now ready to present our first candidate for a generic reducing field of v. 
DEFINITION 3.3. The field of rational functions defined over $k$ of $\operatorname{Proj}_{k}(\mathcal{V})$ is denoted by $\operatorname{proj}_{k}(\mathcal{V})$ and called the projective field of $\mathcal{V}$.

Making use of the terminology explained in $\S \S 1$ and 2, we can now state

THEOREM 3.4. $\operatorname{proj}_{k}(\mathcal{V}) / k$ is a generic reducing field of $\mathcal{V}$. Moreover,

(a) $\operatorname{proj}_{k}(\mathcal{V}) / k$ is a regular field extension.

(b) For every field extension $K / k$, the fields $\operatorname{proj}_{K}\left(\mathcal{V}_{K}\right)$ and $\operatorname{proj}_{k}(\mathcal{V}) \cdot K$ are isomorphic over $K$.

(c) A field extension $K / k$ reduces $\mathcal{V}$ if and only if $\operatorname{proj}_{k}(\mathcal{V}) \cdot K$ is purely transcendental over $K$.

PROOF. Standard facts about rational function fields of algebraic varieties (cf. Proposition 1.4) combined with Lemma 2.1(b) ensure that $\operatorname{proj}_{k}(\mathcal{V}) / k$ is a reducing field of $\mathcal{V}$ satisfying (a), (b). By Lemma 1.5 and Proposition 2.2, the remaining assertions of the theorem will follow as soon as we have shown that, if $\mathcal{V}$ is reduced, $\operatorname{proj}_{k}(\mathcal{V})$ is purely transcendental over $k$. This in turn is a consequence of our next result when specialized to a reduced idempotent $e$.

THEOREM 3.5. Let e be a nonzero idempotent of $\mathcal{V}$. Then $\operatorname{proj}_{k}(\mathcal{V})$ is a purely transcendental extension of $\operatorname{proj}_{k}\left(\mathcal{V}_{2}(e)\right)$.

The proof of this rests on two technicalities, the first one being a rather tedious exercise in Peirce decomposition.

LEMMA 3.6. Let $e$ be a nonzero idempotent of $\mathcal{V}$ and $c$ a reduced idempotent of $\mathcal{V}_{2}(e)$. Then, given elements $u_{i} \in \mathcal{V}_{i}(e)^{+}$for $i=0,1$, the following statements are equivalent.

(i) $u=c^{+}+u_{1}+u_{0}$ has rank one.

(ii) $u_{1} \in \mathcal{V}_{1}(e)^{+} \cap \mathcal{V}_{1}(c)^{+}$and $u_{0}=Q\left(u_{1}\right) c^{-}$.

ProOF. (i) $\Rightarrow\left(\right.$ ii). Clearly, $T\left(u, c^{-}\right)=1$ and hence, by Lemma 3.1(a), $Q(u) c^{-}=$ $u$. Expanding the left-hand side and comparing Peirce components relative to $e$ yields (ii).

(ii) $\Rightarrow$ (i). This is more troublesome. We recall from $[\mathbf{1 4}, \mathrm{JP} 1,12]$ the relations

$$
\begin{gathered}
D(x, y) Q(x)=Q(x) D(y, x), \\
D(x, y) Q(z)+Q(z) D(y, x)=Q(z,\{x y z\}),
\end{gathered}
$$

valid in all Jordan pairs, extend $c$ to a frame $c_{1}=c, c_{2}, \ldots, c_{m}$ of $V_{2}(e)$ and put $d=c_{2}+\cdots+c_{m}$ (so $d=0$ for $m=1$ ). Then $(c, d)$ makes up an orthogonal system of idempotents in $\mathcal{V}$, with Peirce spaces $\mathcal{V}_{\lambda \mu}$ satisfying

$$
V_{2}(e)=V_{11}+V_{12}+V_{22}, \quad V_{1}(e)=V_{01}+V_{02}, \quad V_{0}(e)=V_{00}
$$

Since $u_{0} \in \mathcal{V}_{00}^{+}$and, by hypothesis, $u_{1} \in \mathcal{V}_{01}^{+}$, we may write $u_{0}=u_{00}, u_{1}=u_{01}$. By linearity, we must show

$$
Q(u) v=T(u, v) u \quad\left(v \in \mathcal{V}_{i}(e)^{-}, i=0,1,2\right) .
$$

To this end, we expand the left-hand side to obtain

$$
Q(u) v=Q\left(c^{+}\right) v+Q\left(u_{1}\right) v+Q\left(u_{0}\right) v+\left\{c^{+} v u_{1}\right\}+\left\{c^{+} v u_{0}\right\}+\left\{u_{1} v u_{0}\right\}
$$


observe the trivial relation

$$
x_{11}=T\left(x, c^{-}\right) c^{+} \quad\left(\text { resp. } x_{11}=T\left(c^{+}, x\right) c^{-}\right),
$$

$x_{\lambda \mu}$ being the Peirce- $\lambda, \mu$-component of $x \in \mathcal{V}^{+}$(resp. $\mathcal{V}^{-}$) relative to $(c, d)$, and distinguish the following cases.

Case 1. $i=2$. Then $v=v_{11}+v_{12}+v_{22}$ by (3.5), and (3.6), (3.7), (3.2) yield

$$
Q(u) v=T(u, v) c^{+}+\left\{c^{+} v_{11} u_{01}\right\}+Q\left(u_{01}\right) v_{11} .
$$

Applying (ii) and (3.7) to the last two summands, we obtain

$$
\begin{gathered}
\left\{c^{+} v_{11} u_{01}\right\}=T\left(c^{+}, v\right)\left\{c^{+} c^{-} u_{1}\right\}=T(u, v) u_{1} \\
Q\left(u_{01}\right) v_{11}=T\left(c^{+}, v\right) Q\left(u_{1}\right) c^{-}=T(u, v) u_{0}
\end{gathered}
$$

which settles Case 1 .

Case 2. $i=1$. Then $v=v_{01}+v_{02}$ by (3.5), and (3.6) yields

$$
Q(u) v=\left\{c^{+} v_{01} u_{01}\right\}+\left[Q\left(u_{01}\right) v_{01}+\left\{c^{+} v_{01} u_{00}\right\}\right]+\left\{u_{01} v_{01} u_{00}\right\}
$$

The first summand on the right belongs to $\mathcal{V}_{11}^{+}$, hence (3.7) and (3.1) give

$$
\left\{c^{+} v_{01} u_{01}\right\}=T\left(\left\{c^{+} v_{01} u_{01}\right\}, c^{-}\right) c^{+}=T\left(u_{1},\left\{v_{01} c^{+} c^{-}\right\}\right) c^{+}=T(u, v) c^{+} .
$$

To the term in brackets we apply (ii), (3.4), (3.8) and obtain

$$
\begin{aligned}
Q\left(u_{01}\right) v_{01}+\left\{c^{+} v_{01} u_{00}\right\} & =Q\left(u_{1}\right) v_{01}+D\left(c^{+}, v_{01}\right) Q\left(u_{1}\right) c^{-} \\
& =Q\left(u_{1}\right) v_{01}+Q\left(u_{1},\left\{c^{+} v_{01} u_{01}\right\}\right) c^{-}-Q\left(u_{1}\right) D\left(v_{01}, c^{+}\right) c^{-} \\
& =T(u, v) Q\left(u_{1}, c^{+}\right) c^{-}=T(u, v) u_{1} .
\end{aligned}
$$

Finally, as to the last summand, (ii), (3.3) and (3.1) imply

$$
\begin{aligned}
\left\{u_{01} v_{01} u_{00}\right\} & =D\left(u_{1}, v_{01}\right) Q\left(u_{1}\right) c^{-}=Q\left(u_{1}\right) D\left(v_{01}, u_{1}\right) c^{-} \\
& =Q\left(u_{1}\right)\left\{v_{01} u_{01} c^{-}\right\}=T\left(c^{+},\left\{v_{01} u_{01} c^{-}\right\}\right) Q\left(u_{1}\right) c^{-} \\
& =T\left(\left\{u_{01} c^{-} c^{+}\right\}, v_{01}\right) u_{0}=T(u, v) u_{0},
\end{aligned}
$$

which completes Case 2.

Case 3. $i=0$. Then $v=v_{00}$ by (3.5), and (3.6) yields

$$
Q(u) v=Q\left(u_{01}\right) v_{00}+\left\{u_{01} v_{00} u_{00}\right\}+Q\left(u_{00}\right) v_{00} .
$$

The first summand on the right, being contained in $\mathcal{V}_{11}^{+}$, by (3.7), (3.2), (ii) becomes

$$
Q\left(u_{01}\right) v_{00}=T\left(Q\left(u_{01}\right) v_{00}, c^{-}\right) c^{+}=T\left(Q\left(u_{1}\right) c^{-}, v_{00}\right) c^{+}=T(u, v) c^{+} .
$$

To the second summand we apply (ii), (3.3), (3.9) and conclude

$$
\begin{aligned}
\left\{u_{01} v_{00} u_{00}\right\} & =D\left(u_{01}, v_{00}\right) Q\left(u_{01}\right) c^{-}=Q\left(u_{01}\right) D\left(v_{00}, v_{01}\right) c^{-} \\
& =Q\left(u_{01}\right) D\left(c^{-}, u_{01}\right) v_{00}=D\left(u_{01}, c^{-}\right) Q\left(u_{01}\right) v_{00} \\
& =T(u, v)\left\{u_{1} c^{-} c^{+}\right\}=T(u, v) u_{1},
\end{aligned}
$$

while the last for the same reason transforms to

$$
Q\left(u_{00}\right) v_{00}=Q\left(u_{1}\right) Q\left(c^{-}\right) Q\left(u_{01}\right) v_{00}=T(u, v) Q\left(u_{1}\right) c^{-}=T(u, v) u_{0} .
$$

This settles Case 3 and completes the proof of Lemma 3.6. 
Before turning to the second technicality, we have to introduce some notation. As usual, given a positive integer $n$, we write $\mathbf{A}_{k}^{n}$ for affine $n$-space $\Omega^{n}$, viewed canonically as an algebraic variety defined over $k$. Also, if $X$ denotes a frame in $\bar{V}=V_{\Omega}$, the Conjugacy Theorem 1.1 combined with Lemma 1.3 shows that the dimension of $\bar{V}_{12}(X)^{+}$(resp. $\bar{V}_{01}(X)^{+}$) does not depend on $X$ and, incidentally, will be zero in case $\mathcal{V}$ has degree one (resp. contains invertible elements). Hence we are allowed to call it the coordinate (resp. singular) dimension of $\bar{V}$. Observe now that Theorem 3.4 rests entirely on Theorem 3.5 , which in turn will obviously follow as soon as we have established the following lemma.

LEMMA 3.7. Let e be a nonzero idempotent of $\mathcal{V}$. Then, writing $d$ (resp. $d^{\prime}$ ) for the degree of $\mathcal{V}$ (resp. of $\left.\mathcal{V}_{2}(e)\right)$ and $r$ (resp. s) for the coordinate (resp. singular) dimension of $\mathcal{V}_{\Omega}$, the algebraic varieties

$$
\operatorname{Proj}_{k}(\mathcal{V}) \text { and } \operatorname{Proj}_{k}\left(\mathcal{V}_{2}(e)\right) \times \mathbf{A}_{k}^{s+\left(d-d^{\prime}\right) r}
$$

are birationally equivalent over $k$.

Proof. Setting $\mathcal{W}=V_{2}(e), X=\operatorname{Proj}_{k}(\mathcal{V}), Y=\operatorname{Proj}_{k}(\mathcal{W})$, we must construct a $k$-isomorphism between $k$-open dense subsets of $X$ and $Y \times \mathbf{A}_{k}^{s+\left(d-d^{\prime}\right) r}$. This will be accomplished in several steps.

Step 1. Lemma 3.1(a) shows $Q(v) w=T(v, w) v$ for all $[v] \in Y, w \in \mathcal{W}^{-}$. Using this, we shall exhibit $[v], w$ as above, with $w$ invertible in $\mathcal{W}$, satisfying $T(v, w) \neq 0$. Indeed, if $k$ is infinite, a density argument does the job since $W$ contains no absolute zero divisors. On the other hand, if $k$ is finite, $\mathcal{W}$ must be reduced, so some $v \in \mathcal{W}^{+}$ has $[v] \in Y$, and Lemma 3.1(c) leads to the same conclusion. Now, replacing $e$ by $e^{\prime}=\left(w^{-1}, w\right)$ if necessary and observing the obvious relation $\mathcal{W}=\mathcal{V}_{2}\left(e^{\prime}\right)$, there is no harm in assuming that

$$
X^{\prime}=\left\{[u] \in X: T\left(u, e^{-}\right) \neq 0\right\}, \quad Y^{\prime}=\left\{[v] \in Y: T\left(v, e^{-}\right) \neq 0\right\} \subset X^{\prime}
$$

are ( $k$-open) dense subsets of $X, Y$, respectively.

Step 2. For any $[v] \in Y^{\prime}$ we set

$$
v^{+}=T\left(v, e^{-}\right)^{-1} v, \quad v^{-}=Q\left(e^{-}\right) v^{+}
$$

and conclude from Lemma 3.1(a) combined with $[14,5.2]$ that $c=\left(v^{+}, v^{-}\right)$is a reduced idempotent of $\bar{W}=W_{\Omega}$. Clearly, $v^{ \pm}$is homogeneous of degree 0 in $v$. Also, setting $W=\mathcal{V}_{1}(e)$, the linear map $D\left(v^{+}, v^{-}\right)$acts on $\bar{W}=W_{\Omega}$ as the Peirce 1-projection relative to $c$ since $Q\left(v^{-}\right)$kills $\bar{W}$.

Step 3. We now prove that

$$
Z=\left\{([v], w) \in Y^{\prime} \times \bar{W}:\left\{v^{+} v^{-} w\right\}=w\right\}
$$

is an irreducible subvariety of $Y^{\prime} \times \bar{W}$ defined over $k$ and the map $\alpha: Z \rightarrow X^{\prime}$ given by

$$
\alpha([v], w)=\left[v^{+}+w+Q(w) v^{-}\right]
$$

is a $k$-isomorphism. The first assertion follows from [2, AG (14.15)] since, by Step 2, the rule $([v], w) \mapsto\left([v],\left\{v^{+} v^{-} w\right\}\right)$ determines an idempotent $k$-morphism from $Y^{\prime} \times \bar{W}$ to itself, with image $Z$. As to the second, Lemma 3.6 shows that $\alpha$ does indeed land in $X^{\prime}$ and so is morphism defined over $k$. By comparing Peirce components relative to $e, \alpha$ is easily seen to be injective. Moreover, given $[u] \in X^{\prime}$, 
with Peirce decomposition $u=u_{2}+u_{1}+u_{0}$ relative to $e$, we have $T\left(u_{2}, e^{-}\right)=$ $T\left(u, e^{-}\right) \neq 0$, so $v=u_{2}$ satisfies $[v] \in Y^{\prime}$; also $[u]=\left[v^{+}+v_{1}+v_{0}\right], v_{i}=T\left(u, e^{-}\right)^{-1} u_{i}$ for $i=0,1$, and Lemma 3.6 implies $\left([v], v_{1}\right) \in Z$ as well as $\alpha\left([v], v_{1}\right)=[u]$. Thus $\alpha$ is bijective, with inverse given by $[u] \mapsto\left(\left[u_{2}\right], T\left(u, e^{-}\right)^{-1} u_{1}\right)$, hence must be a $k$-isomorphism.

Step 4. Let $c$ be a reduced idempotent $\overline{\mathcal{W}}$. We set $m=s+\left(d-d^{\prime}\right) r$ and claim

$$
\operatorname{dim}_{k} W=d^{\prime} m, \quad \operatorname{dim}_{\Omega}\left(\overline{\mathcal{V}}_{1}(c) \cap \bar{W}\right)=m \quad\left(\overline{\mathcal{V}}=\mathcal{V}_{\Omega}\right) .
$$

To see this, we extend $c$ to a frame $\left(c_{1}, \ldots, c_{d}\right), c_{1}=c$, of $\overline{\mathcal{V}}$ in such a way that $\left(c_{1}, \ldots, c_{d^{\prime}}\right)$ is a frame of $\overline{\mathcal{W}}$. Writing $\overline{\mathcal{V}}_{\lambda \mu}(0 \leq \lambda, \mu \leq d)$ for the corresponding Peirce components, and setting $I=\left\{1, \ldots, d^{\prime}\right\}$, we obtain

$$
\bar{W}=\sum_{\substack{i \in I \\ j \notin I}} \overline{\mathcal{V}}_{i j}, \quad \overline{\mathcal{V}}_{1}(c) \cap \bar{W}=\sum_{j \notin I} \overline{\mathcal{V}}_{1 j},
$$

and the assertion follows. It is now clear that, for $[v] \in Y^{\prime}$, the map $D_{W}\left(v^{+}, v^{-}\right)$ defined to be the projection $D\left(v^{+}, v^{-}\right.$) acting on $\bar{W}$ (cf. Step 2) has rank $m$.

Step 5 . We put $t=\left(d^{\prime}-1\right) m$ and denote by $\mathbf{G}_{t}(W)$ the Grassmannian of $t$ dimensional subspaces of $\bar{W}$, viewed canonically as a projective algebraic variety defined over $k$. By Step 4, the rule $[v] \mapsto \operatorname{ker} D_{W}\left(v^{+}, v^{-}\right)$defines a $k$-morphism $\beta: Y^{\prime} \rightarrow \mathbf{G}_{t}(W)$. Now pick $\left[v_{0}\right] \in Y^{\prime}$ and let $W^{\prime}$ be a subspace of $W$ having dimension $m$ and satisfying $\beta\left(\left[v_{0}\right]\right) \cap \overline{W^{\prime}}=0$, where $\overline{W^{\prime}}=W_{\Omega}^{\prime}$. Then

$$
\mathcal{U}=\left\{U \in \mathbf{G}_{t}(W): U \cap \overline{W^{\prime}}=0\right\}
$$

is a $k$-open affine piece in $\mathbf{G}_{t}(V)$, and $Y^{\prime \prime}=\beta^{-1}(U)$ is $k$-open dense in $Y$. We now put $Z^{\prime}=Z \cap\left(Y^{\prime \prime} \times \bar{W}\right)$, which is $k$-open dense in $Z$, and contend that

$$
\gamma: Y^{\prime \prime} \times \overline{W^{\prime}} \rightarrow Z^{\prime}, \quad([v], w) \mapsto\left([v],\left\{v^{+} v^{-} w\right\}\right)
$$

is a $k$-isomorphism. Obviously being a well-defined $k$-morphism, we show it is injective by considering $\left(\left[v_{i}\right], w_{i}\right) \in Y^{\prime \prime} \times \overline{W^{\prime}}$ for $i=1,2$ such that $\gamma\left(\left[v_{1}\right], w_{1}\right)=$ $\gamma\left(\left[v_{2}\right], w_{2}\right)$. Then without loss $v_{1}=v_{2}$, which implies $\left\{v_{1}^{+}, v_{1}^{-}, w_{1}-w_{2}\right\}=0$, hence $w_{1}-w_{2} \in \beta\left(\left[v_{1}\right]\right) \cap \overline{W^{\prime}}=0$, as desired. Conversely, let $([v], w) \in Z^{\prime}$. Then $[v] \in Y^{\prime \prime}$, hence $\beta([v]) \cap \overline{W^{\prime}}=0$, and $D_{W}\left(v^{+}, v^{-}\right)$maps $\overline{W^{\prime}}$ bijectively onto im $D_{W}\left(v^{+}, v^{-}\right)$. Therefore, $w$ being contained in the latter, there exists a unique $w_{1} \in \overline{W^{\prime}}$ satisfying $w=\left\{v^{+} v^{-} w_{1}\right\}$. Hence $\gamma$ is altogether bijective, the inverse map being given by $([v], w) \mapsto\left([v],\left(\left.D_{W}\left(v^{+}, v^{-}\right)\right|_{\overline{W^{\prime}}}\right)^{-1} w\right)$. This shows that $\gamma$ is a $k$-isomorphism. Combining Steps 3 and 5 completes the proof of Lemma 3.7. At the same time, Theorems 3.4 and 3.5 have now been safely established.

Replacing $k$ by its algebraic closure in Lemma 3.7, we may specialize $e$ to a reduced idempotent of $\mathcal{V}$, whence $d^{\prime}=1$ and $\operatorname{Proj}_{k}\left(\mathcal{V}_{2}(e)\right)$ collapses to a point. Thus we obtain the following corollary.

COROLLARY 3.8. Let $d$ be the degree and $r$ (resp. s) the coordinate (resp. singular) dimension of $\mathcal{V}_{\Omega}$. Then

$$
\operatorname{dim} \operatorname{Proj}_{k}(\mathcal{V})=s+(d-1) r \text {. }
$$

There is another application of our results which makes it clear why one might be interested in generic reducing fields of minimal transcendence degree. 
COROLLARY 3.9. In the notation of Corollary 3.8 , let $L / k$ be a reducing field of $\mathcal{V}$. Then there is a $k$-imbedding from $\operatorname{proj}_{k}(\mathcal{V})$ to $L$ if and only if $L / k$ has transcendence degree at least $s+(d-1) r$.

ProOF. In view of Corollary 3.8, the existence of a $k$-imbedding $\operatorname{Proj}_{k}(\mathcal{V}) \rightarrow L$ clearly forces $L$ to have transcendence degree at least $s+(d-1) r$. Conversely, let this be so. If $\mathcal{V}$ is reduced, $K=\operatorname{proj}_{k}(\mathcal{V})$ is purely transcendental over $k$, by Theorem 3.4(c), and the assertion again follows from Corollary 3.8. If $\mathcal{V}$ is not reduced, $k$ must be infinite and, again by Theorem $3.4, K \cdot L=\operatorname{proj}_{L}(\mathcal{V} \otimes L)$ is a rational function field over $L$, in $s+(d-1) r$ variables. Since $K$ trivially imbeds into $K \cdot L$ over $k$, the assertion now follows from a lemma of Roquette [24, Lemma 1].

4. The generic norm field of a Jordan pair. A construction of generic splitting fields for associative algebras making use of the generic norm has been given by Heuser [7], Kovacs [13] and Saltman [25]. We wish to pursue this approach here in the Jordan setting and, to this end, require a few preliminaires about Jordan algebras. Accordingly, let $J$ be a unital Jordan algebra over $k$, with $U$-operator $(v, w) \mapsto U_{v} w$. The inversion map, $j$, of $J$ sending $v$ to $v^{-1}$ whenever this makes sense is a birational transformation on $J_{\Omega}$ defined over $k$ whose exact denominator, $N$, normalized to $N(1)=1$, is the generic norm and whose corresponding numerator, \#: $J \rightarrow J, v \mapsto v^{\#}$, is the adjoint of $J$. Note that $N$ is a homogeneous polynomial function whose degree, $d$, by definition is the degree of $J$. Letting $T$ stand for the generic trace of $J$ (defined, up to a sign, as the second logarithmic derivative of $N$ at $\left.1: T=-\left(\mathrm{D}^{2} \log N\right)_{1}\right)$, we record the following well-known formulae $[\mathbf{9}, \mathbf{1 6}, \mathbf{2 7}]$ :

$$
\begin{gathered}
N\left(v^{\#}\right)=N(v)^{d-1}, \\
v^{\# \#}=N(v)^{d-2} v \\
\left(U_{v} w\right)^{\#}=U_{v^{\#}} w^{\#} \\
(\mathrm{D} N)_{v}(w)=T\left(v^{\#}, w\right), \\
(\mathrm{D} j)_{v}(w)=U_{v^{-1}} w \\
N\left(v^{-1}\right)=N(v)^{-1}
\end{gathered}
$$

which imply

$$
v^{-1 \#}=N(v)^{-1} v
$$

Clearing denominators in the relations $U_{v^{-1}} v=v^{-1}, v \circ v^{-1}=2 \cdot 1$, we also have

$$
\begin{aligned}
& U_{v^{\#}} v=N(v) v^{\#}, \\
& v \circ v^{\#}=2 N(v) 1 .
\end{aligned}
$$

PROPOSITION 4.1. Let $J$ be a unital Jordan algebra of degree d over $k$. Then

$$
U_{v} w=T(v, w) v-\frac{1}{N(v)^{d-3}}(\mathrm{D} \#)_{v \#}(w)
$$

for all $v \in J^{\times}, w \in J$. 
PROOF. Differentiating the relation $x^{-1}=N(x)^{-1} x^{\#}$ at $v^{-1}$ gives

$$
\begin{aligned}
U_{v} w & =[\operatorname{by}(4.5)]-(\mathrm{D} j)_{v^{-1}}(w) \\
& =[\operatorname{by}(4.6)]-N(v)^{2}\left\{(\mathrm{D} \#)_{v^{-1}}(w) N(v)^{-1}-(\mathrm{D} N)_{v^{-1}}(w) v^{-1 \#}\right\} \\
& =[\operatorname{by}(4.4)] N(v)^{2} T\left(v^{-1 \#}, w\right) v^{-1 \#}-N(v)(\mathrm{D} \#)_{v^{-1}}(w) \\
& =[\operatorname{by}(4.7)] T(v, w) v-\frac{1}{N(v)^{d-3}}(\mathrm{D \#})_{v^{\#}}(w) .
\end{aligned}
$$

The explicit description of the $U$-operator given in Proposition 4.1 is, of course, well known for $d=2,3[\mathbf{1 0}, \mathbf{1 5}]$, in which cases the right-hand side is a polynomial expression and so the entire formula, being valid in every scalar extension, by density holds for all $v, w \in J$. In full generality, however, Proposition 4.1 seems to arise only implicitly in the literature. Another way to get rid of the denominator which works for arbitrary $d$ consists in replacing $v$ by $v^{\#}$ to obtain

Corollary 4.2. In the situation of Proposition 4.1 we have

$$
U_{v \#} w=T\left(v^{\#}, w\right) v^{\#}-N(v)(\mathrm{D} \#)_{v}(w)
$$

for all $v, w \in J$. In particular, if $v$ is not invertible, $v^{\#}$ has rank at most one.

Here, of course, as in the case of Jordan pairs, the rank of any $v \in J$ is defined as the rank of the linear map $U_{v}$. Also, an idempotent $c$ of $J$ will be called reduced in case $J_{2}(c)=k c \neq 0$. Abbreviating $T(v)=T(v, 1)$ for $v \in J$, we then obtain

LEMMA 4.3. (a) The reduced idempotents in $J$ are precisely the rank one elements having trace 1 .

(b) Suppose $J$ is absolutely simple, let $c$ be a reduced idempotent in $J$, put $J_{0}=$ $J_{0}(c)$ and denote by $N_{0}$ (resp. $\left.\#_{0}\right)$ the generic norm (resp. adjoint) of $J_{0}$. Then

$$
\left(\eta c+v_{0}\right)^{\#}=N_{0}\left(v_{0}\right) c+\eta v_{0}^{\# 0}
$$

for all $\eta \in k, v_{0} \in J_{0}$.

(c) Suppose $J$ is absolutely simple, $v \in J$ is not invertible and satisfies $T\left(v^{\#}\right) \neq$ 0 . Then $c=T\left(v^{\#}\right)^{-1} v^{\#}$ is a reduced idempotents in $\mathrm{J}$, and $v$ is an invertible element of $J_{0}(c)$.

Proof. (a) is immediate by Lemma 3.1(a).

(b) By density it suffices to show this for $v=\eta c+v_{0}$ invertible. As $k c \oplus J_{0}$ is a subalgebra of $J$ having, by absolute simplicity, the same degree, we obtain $N(v)=\eta N_{0}\left(v_{0}\right), v^{-1}=\eta^{-1} c+v_{0}^{\prime}$, where $v_{0}^{\prime}$ is the inverse of $v_{0}$ in $J_{0}$. The assertion follows from this at once.

(c) Since $c$ has trace one, Corollary 4.2 and (a) show that it must be a reduced idempotent in $J$. Then the Peirce projection of $v$ to $J_{0}$ yields

$$
\begin{aligned}
U_{1-c} v & =v-T\left(v^{\#}\right)^{-1} v^{\#} \circ v+T\left(v^{\#}\right)^{-2} U_{v \#} v \\
& =[\text { by }(4.8),(4.9)] v-2 T\left(v^{\#}\right)^{-1} N(v) 1+T\left(v^{\#}\right)^{-2} N(v) v^{\#} \\
& =v .
\end{aligned}
$$

Hence $v \in J_{0}(c)$ and, by (b), $T\left(v^{\#}\right) c=v^{\#}=N_{0}(v) c$. This implies $v \in J_{0}^{\times}$and completes the proof. 
We are now ready to return to our absolutely simple Jordan pair $\mathcal{V}$. Given a maximal idempotent $e$ of $\mathcal{V}$, there exists a(n absolutely simple) Jordan algebra $\mathcal{J}$ over $k$, whose isotopy class, by Proposition 1.2 , only depends on $\mathcal{V}$, such that $V_{2}(e) \cong(J, J)$. Keeping our previous notation, we now obtain

PROPOSITION 4.4. Let $e$ be a maximal idempotent $\mathcal{V}$ and $\mathcal{J}$ a Jordan algebra over $k$ satisfying $V_{2}(e) \cong(J, J)$. Then the collection of singular elements in $J_{\Omega}$, i.e.,

$$
\operatorname{Nor}_{k}(\mathcal{V})=\left\{[v] \in \mathbf{P}_{k}(J): N(v)=0\right\},
$$

makes up an irreducible algebraic hypersurface in $\mathbf{P}_{k}(J)$ defined over $k$ which up to $k$-isomorphism only depends on $\mathcal{V}$ and is called the generic norm surface of $\mathcal{V}$.

ProOF. All this follows trivially from what has been said before and the property of $N$ to be an absolutely irreducible polynomial function, which is a well-known fact, being implied, for example, by the absolute irreducibility of the generic norm of the Jordan pair $\mathcal{V}_{2}(e)[\mathbf{1 4}, 17.3]$.

DEFINITION 4.5 The field of rational functions defined over $k$ of $\operatorname{Nor}_{k}(\mathcal{V})$ is denoted by $\operatorname{nor}_{k}(\mathcal{V})$ and called the generic norm field of $\mathcal{V}$.

THEOREM 4.6. For any maximal idempotent $e$ of $\mathcal{V}$, the generic norm field of $\mathcal{V}$ is purely transcendental over the projective field of $\mathcal{V}_{2}(e)$. In particular, $\operatorname{nor}_{k}(\mathcal{V}) / k$ is a generic reducing field of $\mathcal{V}$.

PrOOF. The second assertion immediately follows from Theorem 3.4 combined with Proposition 2.5. As to the first, there is no harm in assuming that $\mathcal{V}$ itself contains invertible elements and so has the form $\mathcal{V}=(J, J)$, for some absolutely simple Jordan algebra $J$ over $k$. By passing to an appropriate isotope if necessary, we may further assume that $\bar{J}=J_{\Omega}$ contains a reduced idempotent, say $c_{0}$. Then, setting $Y=\operatorname{Proj}_{k}(\mathcal{V})$,

$$
Y_{0}=\{[y] \in Y: T(y) \neq 0\}
$$

contains $\left[c_{0}\right]$ and hence is $k$-open dense in $Y$. Given any reduced idempotent $c$ of $\bar{J}$, we set $\bar{J}(c)=\bar{J}_{2}(c)+\bar{J}_{1}(c)$ and write $E(c)$ for the Peirce projection from $\bar{J}$ to $\bar{J}_{0}(c)$ (so $\operatorname{ker} E(c)=\bar{J}(c)$ ). Observing that $t=\operatorname{dim} \bar{J}(c)$ does not depend on $c$, the $k$-morphism $\varphi: Y_{0} \rightarrow \bar{J}$ defined by $\varphi[y]=T(y)^{-1} y$, whose image, in view of Lemma 4.3(a), consists entirely of reduced idempotents, determines a $k$-morphism $[y] \rightarrow \operatorname{ker} E(\varphi[y])$ from $Y_{0}$ to $\mathbf{G}_{t}(J)$, the Grassmannian of $t$-dimensional subspaces of $\bar{J}$. Hence, choosing a subspace $W$ of $J$ such that $\bar{W}=W_{\Omega}$ is complementary to $\operatorname{ker} E\left(c_{0}\right)$.

$$
Y^{\prime}=\left\{[y] \in Y_{0}: \bar{W} \cap \operatorname{ker} E(\varphi[y])=0\right\}
$$

contains $c_{0}=\varphi\left[c_{0}\right]$ and so is $k$-open dense in $Y$. For pretty much the same reason, setting $X=\operatorname{Nor}_{k}(\mathcal{V})$,

$$
X^{\prime}=\left\{[x] \in X: T\left(x^{\#}\right) \neq 0, \bar{W} \cap \operatorname{ker} E\left(\varphi\left[x^{\#}\right]\right)=0\right\},
$$

the inverse image of $Y^{\prime}$ in $X$ under the adjoint mapping, makes up a $k$-open dense subset of $X$ since, by Lemma 4.3(b), $1-c_{0} \in X^{\prime}$. We now define a $k$-morphism $\Phi: Y^{\prime} \times \mathbf{P}_{k}(W) \rightarrow X$ by $\Phi([y],[w])=[E(\varphi[y]) w]$ and contend that $Z=\Phi^{-1}\left(X^{\prime}\right)$ is $k$-open dense in $Y^{\prime} \times \mathbf{P}_{k}(W)$. Of course, we need only show $Z \neq \varnothing$. To see this, we note that $E\left(c_{0}\right)$ maps $\bar{W}$ bijectively onto $\bar{J}_{0}=\bar{J}_{0}\left(c_{0}\right)$, so some $w \in \bar{W}$ has 
$x=E\left(c_{0}\right) w$ invertible in $\bar{J}_{0}$. By Lemma $4.3(\mathrm{~b})$ this forces $x^{\#}=N_{0}(x) c_{0}$ hence $T\left(x^{\#}\right)=N_{0}\left(x_{0}\right) \neq 0$, and $\varphi\left[x^{\#}\right]=c_{0}$. Altogether, we have $\left(\left[c_{0}\right],[w]\right) \in Z$, as claimed, and obtain an induced $k$-morphism

$$
\Phi: Z \rightarrow X^{\prime}, \quad([y],[w]) \mapsto \Phi([y],[w])=[E(\varphi[y]) w] .
$$

Conversely, it is straightforward to check that the $k$-morphism $\Psi: X^{\prime} \rightarrow Y^{\prime} \times \mathbf{P}_{k}(W)$ defined by

$$
\Psi([x])=\left(\left[x^{\#}\right],\left[\left\{\left.E\left(\varphi\left[x^{\#}\right]\right)\right|_{\bar{W}}\right\}^{-1} x\right]\right)
$$

makes sense in view of Lemma $4.3(\mathrm{c})$, satisfies $\Phi \circ \Psi=$ id and so may be viewed as a $k$-morphism $\Psi: X^{\prime} \rightarrow Z$. Once we have also shown $\Psi \circ \Phi=$ id, it will follow that $\Phi: Z \rightarrow X^{\prime}$ is a $k$-isomorphism, forcing $Y \times \mathbf{P}_{k}(W)$ and $X$ to be birationally equivalent over $k$, and we are done.

Now, given $([y],[w]) \in Z$, we have

$$
\Psi(\Phi([y],[w]))=\Psi([E(\varphi[y]) w])=\left(\left[y^{\prime}\right],\left[w^{\prime}\right]\right),
$$

where, observing $E(\varphi[y])=U_{1-\varphi[y]}$,

$$
\begin{aligned}
{\left[y^{\prime}\right] } & =\left[\left\{U_{1-\varphi[y]} w\right\}^{\#}\right]=[\text { by }(4.3)]\left[U_{\left.(1-\varphi[y])^{\#} w^{\#}\right]}\right. \\
& =[\text { by Lemma 4.3(b) }]\left[U_{\varphi[y]} w^{\#}\right] \\
& =[\text { by Lemma 3.1(a) }][\varphi[y]]=[y],
\end{aligned}
$$

hence

$$
\left[w^{\prime}\right]=\left[\left\{\left.E\left(\varphi\left[y^{\prime}\right]\right)\right|_{\bar{W}}\right\}^{-1} E(\varphi[y]) w\right]=[w] .
$$

Because of Theorem 4.6, it is now clear that not only parts (a) and (b) but also (c) of Theorem 3.4 mutatis mutandis carry over to $\operatorname{nor}_{k}(\mathcal{V})$ in place of $\operatorname{proj}_{k}(\mathcal{V})$. We omit the details.

5. Examples. In this section, we wish to identify the generic reducing fields introduced before, as well as their associated algebraic varieties, for the standard examples of absolutely simple Jordan pairs. In view of Proposition 2.4, we may confine ourselves to Jordan pairs with invertible elements, having the form $(J, J)$ for some unital Jordan algebra $J$. As a matter of notation, we do not always distinguish carefully between $J$ and $(J, J)$; for example, we write $\operatorname{Proj}_{k}(J)$ rather than $\operatorname{Proj}_{k}(J, J)$ and call this the projective variety of $J$.

EXAMPLE 5.1 Let $V$ be a vector space of dimension $n>2$ over $k, q: V \rightarrow k$ an absolutely nondegenerate quadratic form and $\mathcal{V}=\mathcal{V}(q)$ (cf. Example 2.7). Then it is easily checked that a nonzero element of $V$ has rank one if and only if it is isotropic relative to $q$. On the other hand, if we choose an anisotropic vector $e^{+} \in V$ and put $e^{-}=q\left(e^{+}\right)^{-1} e^{+}$, then $e=\left(e^{+}, e^{-}\right)$is a maximal idempotent, and the rule $(v, w) \rightarrow\left(v, q\left(e^{+}\right) \bar{w}\right)$ determines an isomorphism from $\mathcal{V}=\mathcal{V}_{2}(e)$ onto the Jordan pair $(J, J)$, where $J$ is the Jordan algebra of the quadratic form $\left(V, q\left(e^{+}\right)^{-1} q, e^{+}\right)$ with base point and $w \mapsto \bar{w}$ stands for the canonical involution of $J$. Hence, as $q\left(e^{+}\right)^{-1} q$ is the generic norm of $\mathrm{J}$,

$$
\operatorname{Proj}_{k}(\mathcal{V})=\operatorname{Nor}_{k}(\mathcal{V}), \quad \operatorname{proj}_{k}(\mathcal{V})=\operatorname{nor}_{k}(\mathcal{V}),
$$

and the latter is nothing else than the generic zero field $k(q)_{0}$ of $q$ constructed by Knebusch [11, p. 71 and Theorem 3.3]. Also $\operatorname{dim} \operatorname{Proj}_{k}(\mathcal{V})=n-2$. 
EXAMPLE 5.2. Let $A$ be a central simple associative algebra of degree $d$ and dimension $d^{2}$ over $k$. We write $\mathcal{V}=\mathcal{V}(A)$ for the associated Jordan pair (cf. Example 2.6) and $A^{+}$for the associated Jordan algebra, so $\mathcal{V}=\left(A^{+}, A^{+}\right)$. Clearly,

$$
\operatorname{Nor}_{k}\left(A^{+}\right)=\operatorname{Nor}_{k}(A), \quad \operatorname{nor}_{k}\left(A^{+}\right)=\operatorname{nor}_{k}(A),
$$

i.e., the generic norm surface (field) of $A^{+}$agrees with the analogous object attached to $A$, which has been studied by Heuser [7] and, in the affine setting, by Kovacs [13] and Saltman [25]. We now wish to clarify the relation between the projective field of $\mathcal{V}$ and the generic splitting field of $A$ constructed by Amitsur [1]. For want of a convenient reference, let us briefly indicate an ad hoc treatment of the latter's relationship to Brauer-Severi varieties.

Recall [26, X, $\S 6$, p. 168] that a $k$-variety $X$ is said to be Brauer-Severi in case there exists a separable algebraic field extension $l / k$ such that $X$ becomes $l$-isomorphic to full projective space. Now, arguing pretty much the same way as in the proof of Theorem 3.2, for example, the set $\operatorname{Br}_{k}(A)$ of minimal left ideals in $A_{\Omega}$ is easily seen to be an irreducible subvariety over $k$ of the Grassmannian of $d$-dimensional subspaces of $A_{\Omega}$. If $A$ is split and so identifies with $\mathrm{M}_{d}(k)$, the map

$$
\mathbf{P}_{k}^{d-1} \rightarrow \operatorname{Br}_{k}(A), \quad[a] \mapsto A_{\Omega} a^{t} a \quad\left({ }^{t} a=\text { transpose of } a\right)
$$

turns out to be $k$-isomorphism, with inverse

$$
\operatorname{Br}_{k}(A) \rightarrow \mathbf{P}_{k}^{d-1}, \quad I \mapsto \operatorname{im}^{t} s
$$

where $s$ is any nonzero element of $I \in \mathrm{Br}_{k}(A)$, and ${ }^{t} s$ acts canonically on (column space) $\Omega^{d}$. Returning to the general case where $A$ is an abitrary central simple associative algebra, it now follows that $\operatorname{Br}_{k}(A)$ is indeed a Brauer-Severi variety. Also, $\operatorname{br}_{k}(A)$, the field of rational functions on $\operatorname{Br}_{k}(A)$ defined over $k$, enjoys the following remarkable property (cf. [1, Theorem 9.1]), reminiscent of Theorem 3.4(c): $A$ field extension $l / k$ splits $A$ if and only if $\mathrm{br}_{k}(A) \cdot l$ purely transcendental over $l$; in particular, $\operatorname{br}_{k}(A)$ is a generic splitting field of $A$, and actually the one constructed by Amitsur. Following Roquette [23], we call $\mathrm{br}_{k}(A)$ the Brauer field of $A$. We obviously have $\operatorname{dim} \mathrm{Br}_{k}(A)=d-1$. By contrast, Corollary 3.8 yields

$$
\operatorname{dim} \operatorname{Proj}_{k}\left(A^{+}\right)=2(d-1) \text {. }
$$

We can now prove

PROPOSITION 5.3. Let $A$ be a central simple associative algebra over $k$. Then the algebraic $k$-varieties

$$
\operatorname{Proj}_{k}\left(A^{+}\right) \text {and } \mathrm{Br}_{k}(A) \times \mathrm{Br}_{k}\left(A^{\text {op }}\right)
$$

are isomorphic. In particular, $\operatorname{proj}_{k}\left(A^{+}\right)$is the free composite $\operatorname{br}_{k}(A) \cdot \operatorname{br}_{k}\left(A^{\mathrm{op}}\right)$ and purely transcendental over $\operatorname{br}_{k}(A)$.

PROOF. It is readily checked that the map

$$
\operatorname{Proj}_{k}\left(A^{+}\right) \rightarrow \operatorname{Br}_{k}(A) \times \operatorname{Br}_{k}\left(A^{\text {op }}\right), \quad[u] \mapsto\left(A_{\Omega} u, u A_{\Omega}\right)
$$

is a $k$-isomorphism, with inverse sending $(I, J) \in \mathrm{Br}_{k}(A) \times \mathrm{Br}_{k}\left(A^{\text {op }}\right)$ to $J I$, which proves the first assertion. Using Proposition 1.4, we conclude

$$
\operatorname{proj}_{k}\left(A^{+}\right) \cong \operatorname{br}_{k}(A) \cdot \operatorname{br}_{k}\left(A^{\text {op }}\right) \cong \operatorname{br}_{k}\left(A^{\text {op }}\right) \cdot \operatorname{br}_{k}(A) \text {. }
$$

But $K=\operatorname{br}_{k}(A)$ splits $A$, hence $A^{\text {op }}$, and Example 5.2 shows that $\operatorname{proj}_{k}\left(A^{+}\right)$is purely transcendental over $K$. 
COROLlARY 5.4 (Saltman [25, Theorem 4.2]). The generic norm field of a central simple associative algebra is purely transcendental over its Brauer field.

ProOF. This follows immediately from Proposition 5.3 and Theorem 4.6.

Next we turn to pairs $(A, *)$ where $A$ is a simple associative $k$-algebra, $*$ is a $k$-involution on $A$ and $(A, *)$ is central in the sense that the set of elements in $l$, the center of $A$, fixed under $*$ agrees with $k$. Consider the Jordan pair associated with the Jordan algebra $\mathrm{H}(A, *)$ of $*$-symmetric elements of $A$. The case when $*$ is an involution of the second kind is not particulary interesting here since this implies that $l / k$ is a separable quadratic extension and, by Theorem $3.4, \operatorname{proj}_{k}(\mathrm{H}(A, *)) \otimes$ $l \cong \operatorname{proj}_{l}\left(A^{+}\right)$, the latter field being explicitly related to the Brauer field of $A$ by Proposition 5.3. A similar remark applies to the generic norm field. In the sequel, we therefore focus attention on involutions of the first kind.

EXAMPLE 5.5. Let $(A, *)$ be a central simple associative $k$-algebra with involution of the first kind and of orthogonal type. As before, write $d$ for the degree of $A$. Standard results from Jordan theory imply that the (generic) reducing fields of $\mathrm{H}(A, *)$ (more precisely, of the Jordan pair associated with it) are the same as the (generic) splitting fields of $A$. In particular, because of Theorem 4.6, we recover Jacobson's result $[8,4.19]$, according to which the generic norm field of $\mathrm{H}(A, *)$ is a generic splitting field of $A$. Also, in conjunction with the formula

$$
\operatorname{dim} \operatorname{Proj}_{k}(\mathrm{H}(A, *))=d-1 \text {, }
$$

being implied by Corollary 3.8, one naturally expects that the projective field of $\mathrm{H}(A, *)$ is intimately tied up with the Brauer field of $A$. Our next result confirms this expectation.

Proposition 5.6. Let $(A, *)$ be a central simple associative $k$-algebra of degree $d$ with involution of the first kind and of orthogonal type. Then the k-varieties $\operatorname{Proj}_{k}(\mathrm{H}(A, *))$ and $\mathrm{Br}_{k}(A)$, hence also the field extensions $\operatorname{proj}_{k}(\mathrm{H}(A, *)) / k$ and $\operatorname{br}_{k}(A) / k$, are isomorphic.

Proof. The map

$$
\operatorname{Proj}_{k}(\mathrm{H}(A, *)) \rightarrow \mathrm{Br}_{k}(A), \quad[u] \mapsto A_{\Omega} u
$$

is easily seen to be a $k$-isomorphism, with inverse sending $I \in \operatorname{Br}_{k}(A)$ to $I^{*} I$.

EXAMPLE 5.7 Let $(A, *)$ be a central simple associative $k$-algebra of degree $d$ with involution of the first kind and of symplectic type. Then $d$ is even. Rather than looking at $\mathrm{H}(A, *)$, we prefer to look at $\mathrm{H}^{\prime}(A, *)$, the outer ideal of $\mathrm{H}(A, *)$ generated by 1 , which agrees with $\mathrm{H}(A, *)$ for char $k \neq 2$. Again by standard results from Jordan theory, the (generic) reducing fields of $\mathrm{H}^{\prime}(A, *)$ are the same as the (generic) $\frac{1}{2}$-splitting fields of $A$ in the sense of Jacobson [8], i.e., the field extensions $K / k$ with $A_{K}$ having Schur index at most 2 . Hence from our previous results we recover $[8,4.29]$ that the generic norm field of $\mathrm{H}^{\prime}(A, *)$ is a generic $\frac{1}{2}$-splitting field of $A$. An analogous remark applies to the projective field of $\mathrm{H}^{\prime}(A, *)$. Note that Corollary 3.8 implies

$$
\operatorname{dim} \operatorname{Proj}_{k}\left(\mathrm{H}^{\prime}(A, *)\right)=2(d-2) .
$$

EXAMPLE 5.8. Let $J$ be a central simple exceptional Jordan algebra over $k$. Then $J$ has dimension 27, hence $\operatorname{dim} \operatorname{Nor}_{k}(J)=25$. Also, Corollary 3.8 yields 
$\operatorname{dim} \operatorname{Proj}_{k}(J)=16 . \operatorname{Proj}_{k}(J)$ is, of course, nothing else than the classical octonion plane studied by many authors from various points of view. The reader is referred to Faulkner [4] for a brief summary of the historical development. The field extensions nor $_{k}(J) / k$ and $\operatorname{proj}_{k}(J) / k$, by Theorems 3.4 and 4.6, are clearly irrelevant if $J$ is reduced. On the other hand, in the general situation, a proper understanding of them may conceivably be linked to the isomorphism problem for division algebras, which is still in a rather unsatisfactory state at present (see $[20,22]$ ).

6. An application to exceptional Jordan division algebras. Professor J. C. Ferrar has raised the question as to whether two nonisomorphic central exceptional Jordan division algebras over $k$ can be distinguished from one another by finding an extension field which splits (resp. reduces) one without splitting (resp. reducing) the other. In spite of the fact that the analogous problem on the level of composition algebras has been settled affirmatively by Ferrar himself $[4$, Theorem 2], we shall now give a negative answer to this question. In order to do so, we briefly describe the first Tits construction of exceptional simple Jordan algebras. Let $A$ be a central simple associative algebra of degree 3 over $k$ and $\lambda \in k^{\times}$. Then the generic norm $N$, the adjoint \# and the generic trace $T$ of $A$, which agree with the corresponding notions in the associated Jordan algebra $A^{+}$(see $\S 4$ ), extend to the vector space $V=A \oplus A \oplus A$ according to the rules

$$
\begin{aligned}
N(v) & =N\left(a_{0}\right)+\lambda N\left(a_{1}\right)+\lambda^{-1} N\left(a_{2}\right)-T\left(a_{0} a_{1} a_{2}\right), \\
v^{\#} & =\left(a_{0}^{\#}-a_{1} a_{2}, \lambda^{-1} a_{2}^{\#}-a_{0} a_{1}, \lambda a_{1}^{\#}-a_{2} a_{0}\right), \\
T(v, w) & =T\left(a_{0}, b_{0}\right)+T\left(a_{1}, b_{2}\right)+T\left(a_{2}, b_{1}\right)
\end{aligned}
$$

for $v=\left(a_{0}, a_{1}, a_{2}\right), w=\left(b_{0}, b_{1}, b_{2}\right)$. Note that \# is a quadratic mapping and write $(v, w) \mapsto v \times w$ for its bilinearization. McCrimmon [15] has shown that the identity $(1,0,0) \in V$ and the $U$-operator $U_{v} w=T(v, w) v-v^{\#} \times w$ (cf. Proposition 4.1 with $d=3$ ) give $V$ the structure of a unital quadratic Jordan algebra, written as $J(A, \lambda)$ and said to arise from $A, \lambda$ by means of the first Tits construction. $J(A, \lambda)$ is a Jordan division algebra if and only if ( $A$ is an associative division algebra and) $\lambda$ is not a generic norm of $A[\mathbf{1 5}$, Theorem 6]; otherwise $J(A, \lambda)$ is not only reduced but actually split [21, Corollary 4.2], so, in this special case, splitting fields and reducing fields are the same. We can now prove the following theorem.

THEOREM 6.1. Let $A$ be a central simple associative algebra of degree 3 over $k$ and suppose $\lambda \in k^{\times}$is not a generic norm of $A$. Then the exceptional Jordan division algebras $J(A, \lambda)$ and $J\left(A^{\mathrm{op}}, \lambda\right)$ have the same splitting fields but, in general, are not isomorphic.

Proof. To establish the first part, it suffices to show, according to Theorem 4.6, that the generic form fields of $J=J(A, \lambda)$ and $J^{\prime}=J\left(A^{\text {op }}, \lambda\right)$ are $k$-isomorphic, or, what amounts to the same, that the corresponding generic norm surfaces, $X=$ $\operatorname{Nor}_{k}(J)$ and $X^{\prime}=\operatorname{Nor}_{k}\left(J^{\prime}\right)$, are birationally equivalent over $k$. To see this, we note that

$$
U=\left\{\left[\left(a_{0}, a_{1}, a_{2}\right)\right] \in X: N\left(a_{1}\right) \neq 0\right\},
$$

is $k$-open dense in $X$ since, for example, $\left(0,1,-\sqrt[3]{\lambda^{2}}\right) \in U$. Similarly,

$$
U^{\prime}=\left\{\left[\left(a_{0}, a_{1}, a_{2}\right)\right] \in X^{\prime}: N\left(a_{1}\right) \neq 0\right\}
$$


is $k$-open dense in $X^{\prime}$. Now the rule

$$
\left(a_{0}, a_{1}, a_{2}\right) \mapsto\left(a_{0}, a_{1}, a_{1} a_{2} a_{1}^{-1}\right)
$$

determines a birational transformation $\varphi$ defined over $k$ from $\mathbf{P}_{k}(J)$ to $\mathbf{P}_{k}\left(J^{\prime}\right)$. Also, observing (6.1) as well as

$$
T\left(a_{0} a_{1} a_{2}\right)=T\left(a_{1} a_{2} a_{1}^{-1} a_{1} a_{0}\right),
$$

and writing $N, N^{\prime}$ for the generic norm of $J, J^{\prime}$, respectively, it is readily checked that

$$
N^{\prime}\left(\left(a_{0}, a_{1}, a_{1} a_{2} a_{1}^{-1}\right)\right)=N\left(\left(a_{0}, a_{1}, a_{2}\right)\right)
$$

whenever this makes sense. Hence $\varphi$ induces a $k$-isomorphism $U \rightarrow U^{\prime}$, yielding the first part of the theorem. With regard to the second, it is an open question as to whether $J$ and $J^{\prime}$ are always nonisomorphic. Specific examples for this kind of situation arise as follows. Let $k$ be a local field, $\pi$ a prime element of its valuation ring and $D$ an unramified central associative division algebra of degree 3 over $k$. Then $J\left(D^{\text {op }}, \pi\right)$ and $J\left(D, \pi^{2}\right)$ become isomorphic under the transformation

$$
\left(a_{0}, a_{1}, a_{2}\right) \mapsto\left(a_{0}, \pi^{-1} a_{2}, \pi a_{1}\right),
$$

hence $\left[\mathbf{1 7}\right.$, Theorem 4] shows that $J(D, \pi)$ and $J\left(D^{\mathrm{op}}, \pi\right)$ cannot be isomorphic.

\section{REFERENCES}

1. S. A. Amitsur, Generic splitting fields of central simple algebras, Ann. of Math. (2) 62 (1955), 8-43.

2. A. Borel Linear algebraic groups, Benjamin, New York and Amsterdam, 1969.

3. N. Bourbaki, Eléments de mathématique, Algèbre Commutative, Hermann, Paris, 1969.

4. J. R. Faulkner, Octonion planes defined by quadratic Jondan algebras, Mem. Amer. Math. Soc. No. 104 (1970).

5. J. C. Ferrar, Generic splitting fields of composition algebras, Trans. Amer. Math. Soc. 128 (1967), 506-514.

6. K.-H. Helwig and U. Hirzebruch, Über reguläre Jordan-Algebren und eine Äquivalenzrelation von M. Koecher, Indag. Math. 30 (1968), 461-465.

7. A. Heuser, Über den Funktionenkörper der Normfläche einer zentral einfachen Algebra, J. Reine Angew. Math. 301 (1978), 105-113.

8. N. Jacobson, Some applications of Jordan norms to involutorial simple associative algebras, Adv. in Math. 48 (1983), 149-165.

9. N. Jacobson and J. Katz, Generically algebraic quadratic Jordan algebras, Scripta Math. 29 (1973), 215-227.

10. N. Jacobson and K. McCrimmon, Quadratic Jordan algebras of quadratic forms with base point, J. Indian Math. Soc. (N.S.) 35 (1971), 1-45.

11. M. Knebusch, Generic splitting of quadratic forms. I, Proc. London Math. Soc. (3) 33 (1976), 65-93.

12. __ Generic splitting of quadratic forms. II, Proc. London Math. Soc. (3) 34 (1977), 1-31.

13. A. Kovacs, Generic splitting fields, Comm. Algebra 6 (1978), 1017-1035.

14. O. Loos, Jordan pairs, Lecture Notes in Math., vol. 460, Springer-Verlag, Berlin, Heidelberg and New York, 1975.

15. K. McCrimmon, The Freudenthal-Springer-Tits constructions of exceptional Jordan algebras, Trans. Amer. Math. Soc. 139 (1969), 495-510.

16. - The generic norm of an isotope of a Jordan algebra, Scripta Math. 29 (1973), 229-236.

17. H. P. Petersson, Exceptional Jondan division algebras over a field with a discrete valuation, J. Reine Angew. Math. 274/275 (1975), 1-20.

18. __ Zur Arithmetick der Jordan-Paare, Math. Z. 147 (1976), 139-161.

19. __ Conjugacy of idempotents in Jordan pairs, Comm. Algebra 6 (1978), 673-715. 
20. H. P. Petersson and M. L. Racine, Classificiation of Jordan algebras arising from the Tits process, J. Algebra (to appear).

21. __ Springer forms and the first Tits construction of exceptional Jordan division algebras, Manuscripta Math. 45 (1984), 249-272.

22. __ Pure and generic first Tits constructions of exceptional Jordan division algebras (to appear).

23. P. Roquette, On the Galois cohomology of the projection linear group and its applications to the construction of generic splitting fields of algebras, Math. Ann. 150 (1963), 411-439.

24. __ Isomorphisms of generic splitting fields of simple algebras, J. Reine Angew. Math. 214/215 (1964), 207-226.

25. D. Saltman, Norm polynomials and algebras, J. Algebra 62 (1980), 333-345.

26. J. P. Serre, Corps locaux, Hermann, Paris, 1968.

27. T. A. Springer, Jordan algebras and algebraic groups, Ergeb. Math. Grenzgeb., vol. 75, SpringerVerlag, Berlin, Heidelberg and New York, 1973.

28. E. Witt, Über ein Gegenbeispiel zum Normensatz, Math. Z. 39 (1934), 462-467.

FACHBEREICH MATHEMATIK DER FERnUNIVERSität, LÜtzoWstrasse 125, D-58 HAGEN, BUNDESREPUBLIK DEUTSCHLAND 